\title{
If the Redshift Depends on the Pressure then the Acceleration of the Universe Can Be Explained
}

\author{
Victor R. Krym \\ Department of Mathematics and Mechanics, St.-Petersburg State University, St.-Petersburg, Russia \\ Email: vkrym12@rambler.ru
}

Received May 16, 2013; revised June 19, 2013; accepted July 22, 2013

Copyright (C) 2013 Victor R. Krym. This is an open access article distributed under the Creative Commons Attribution License, which permits unrestricted use, distribution, and reproduction in any medium, provided the original work is properly cited.

\begin{abstract}
We propose a model where the Hubble's law is slightly changed. We propose new interpretation of the covariant divergence of the energy-impulse vector and this produce a new correction to redshift. Acceleration of the expansion of the Universe appeared as a pure observational effect. High values of the mass density are consistent with the experimental data on Supernova Ia within this FRW model without the cosmological constant $(\Lambda=0)$.
\end{abstract}

Keywords: Relativistic Cosmology; FRW Models; Section Curvature; The Problem of Matter Creation; The Continuity Equation

\section{Introduction}

The equation of continuity with the classical linear equation of state $P=(\gamma-1) \rho[1-6]$ where $P$ is the pressure, $\rho$ is the density of matter and $w=\gamma-1$ is the coefficient, leads to negative pressure values for $w$ $(w<0)$. The $\Lambda$ CDM model predicts effective value for $w$ which is negative $[7,8]$. Then for a small scale factor $a$ in the beginning of the Universe the total amount of matter in the Universe would be negligible. When the scale factor increased the matter appeared literally from nothing.

We propose new correction to redshift which can be useful in cosmology. This explains the appeared acceleration of the Universe. High values of the mass density are consistent with the experimental data on Supernova Ia within this model without the cosmological constant $(\Lambda=0)$. We compared this model with the experimental data of the Supernova Cosmology Project Supernova Ia compilation. We assumed that $\Omega_{0 m}=1.01$ and $\Lambda=0$, hence $\Omega_{0 \kappa}=1-\Omega_{0 m}=-0.01$ (yet $\kappa>0$ and curvature is positive). We assumed also that $w(z)=v z$, where $v$ is the parameter of this model and $z$ is the redshift. We found the optimal values

$$
v=-0.05_{ \pm 0.02}
$$

and

$$
H_{0}=66_{ \pm 5} \frac{\mathrm{km}}{\mathrm{s} \cdot \mathrm{Mps}} .
$$

The quality of this regression was as high as it was in the $\Lambda \mathrm{CDM}$ model. Yet the value of $w$ was much less in the absolute value than in the $\Lambda \mathrm{CDM}$ model. The acceleration of the Universe appeared to be a pure observational effect due to the negative pressure.

\section{The Equation of Continuity}

The Einstein equations with the cosmological constant $\Lambda$ are

$$
R_{\alpha \beta}-\frac{1}{2} R g_{\alpha \beta}=\varkappa T_{\alpha \beta}+\Lambda g_{\alpha \beta}, \quad \alpha, \beta=0, \cdots, 3,
$$

where

$$
\varkappa=\frac{8 \pi G_{N}}{c^{2}}=1.8659218 \times 10^{-26} \mathrm{~m} / \mathrm{kg}
$$

- the Einstein gravitational constant [9, p. 347],

$$
G_{N}=6.6725985 \times 10^{-11} \mathrm{~m}^{3} /\left(\mathrm{kg} \cdot \mathrm{c}^{2}\right)
$$

- the Newton gravitational constant.

To solve these equations we should know the energymomentum tensor $T_{\alpha \beta}$. For synchronous coordinates the energy-momentum tensor is

$$
\left(T_{\beta}^{\alpha}\right)_{\alpha, \beta=0, \cdots, 3}=\operatorname{Diag}(\rho,-P,-P,-P),
$$

where $\rho$ is the density of matter and $P$ is the pressure of matter. The covariant divergence of the energymomentum tensor is zero: 


$$
\sum_{\alpha=0}^{3} \nabla_{\alpha} T_{\beta}^{\alpha}=0, \quad \beta=0, \cdots, 3
$$

If

$$
\beta=0, \quad \sum_{\alpha=0}^{3} \nabla_{\alpha} T_{0}^{\alpha}=\dot{\rho}+(\rho+P) \sum_{s=1}^{3} \Gamma_{0 s}^{s} .
$$

Considering (22) we obtain

$$
\dot{\rho}+3 \frac{\dot{a}}{a}(\rho+P)=0
$$

for the $T_{0}^{s}$ component. Assume that the pressure is proportional to the density:

$$
P=(\gamma-1) \rho .
$$

for the dust matter this value is $\gamma=1$ and the value for radiation is $\gamma=4 / 3[9$, p. $\sim 123]$. Therefore

$$
\dot{\rho}+3 \gamma \frac{\dot{a}}{a} \rho=0
$$

and

$$
\rho=c_{1} a^{-3 \gamma} .
$$

Considering physical dimensions of values, the Equation (3) is better as

$$
\rho / \rho_{0}=\left(a / a_{0}\right)^{-3 \gamma} .
$$

Hence

$$
c_{1}=\rho_{0} a_{0}^{3 \gamma} .
$$

Let us compare this equation with the change of volume of the Universe for closed models. The FriedmannRobertson-Walker metric in the cosmic time and in the polar coordinates is

$$
\begin{aligned}
(\mathrm{d} s)^{2} & =(\mathrm{d} t)^{2} \\
& -a(t)^{2}\left(\frac{(\mathrm{d} r)^{2}}{1-\kappa r^{2}}+r^{2}\left((\mathrm{~d} \theta)^{2}+\sin ^{2} \theta(\mathrm{d} \phi)^{2}\right)\right),
\end{aligned}
$$

where

$$
0 \leq r \leq 1 / \sqrt{\kappa}, \quad 0 \leq \theta \leq \pi, \quad 0 \leq \varphi<2 \pi
$$

and $\kappa$ is the curvature parameter $(\kappa>0)$. The 3 -volume element at $t=$ const is

$$
\sqrt{-\operatorname{det} g}=a(t)^{3} r^{2} / \sqrt{1-\kappa r^{2}} \sin \theta \mathrm{d} r \mathrm{~d} \theta \mathrm{d} \varphi .
$$

The volume of the Universe at $t=$ const is

$$
V=\int_{0}^{1 / \sqrt{\kappa}} \int_{0}^{\pi} \int_{0}^{2 \pi} \frac{a^{3} r^{2}}{\sqrt{1-\kappa r^{2}}} \sin \theta \mathrm{d} r \mathrm{~d} \theta \mathrm{d} \varphi=\frac{\pi^{2} a^{3}}{\kappa^{3 / 2}} .
$$

Since our Universe is homogeneous, the total amount of matter in the Universe at some $t=$ const is

$$
\rho V=c_{1} a^{-3 \gamma} \frac{\pi^{2} a^{3}}{\kappa^{3 / 2}}=c_{1} \frac{\pi^{2} a^{3-3 \gamma}}{\kappa^{3 / 2}}=c_{1} \frac{\pi^{2} a^{-3 w}}{\kappa^{3 / 2}},
$$

where $w=\gamma-1$. Hence for $w<0$ and $a$ sufficiently small the total amount of matter in the Universe will be negligible: $\rho V \rightarrow 0$ when $a \rightarrow 0$ and $w<0$. Cosmology with $w \neq 0$ violates the law of conservation of matter (conservation of leptonic and baryonic numbers [10]). The idea that matter originated from radiation is not a good idea because $c^{2}$ is too large. The matter antimatter asymmetry also cannot be explained in this way. Cosmology with negative pressure $w<0$ contained a "smooth bounce" from the collapsing to expanding stage and this was also due to the same fact. Since the matter disappeared, the gravitation force was negligible and we obtained the smooth bounce with the horizontal tangent [11-13]. The only equation which is fully consistent with (5) and conservation of leptonic and baryonic numbers is

$$
\frac{\rho}{\rho_{0}}=\left(\frac{a}{a_{0}}\right)^{-3} .
$$

This is due to the equation $\rho V=$ const. The Equation (3) could work for the appropriate epoch only.

Let us try to interpret the covariant divergence of the $\left(p^{0}, \boldsymbol{j}\right)$ 4-vector. If currents are negligible (as in the comoving frame), then

$$
\frac{\partial p^{0}}{\partial t}
$$

will get an additional nonzero component and $p^{0}$ will increase or decrease additionally. It seems reasonable because $p^{0}$ is the energy and its change is the redshift. Hence this equation should give a contribution to the redshift (and not bound the matter components). Since

$$
\sum_{s=0}^{3} \nabla_{s} T_{0}^{s}=\dot{\rho}+3 \frac{\dot{a}}{a}(\rho+P)
$$

and

$$
\rho / \rho_{0}=\left(a / a_{0}\right)^{-3}
$$

due to (5), this covariant divergence is

$$
\sum_{s=0}^{3} \nabla_{s} T_{0}^{s}=3 \frac{\dot{a}}{a} P
$$

where $P$ is the pressure. Assume that

$$
3 \frac{\dot{a}}{a} P=-\frac{\partial}{\partial t} \frac{\delta p_{0}}{\delta V} .
$$

Here

$$
\rho=E=\frac{\delta p_{0}}{\delta V}
$$

is the energy density per unit volume. Integrating

$$
3 \frac{\dot{a}}{a} P / \rho=-E^{\prime} / E
$$


with $P / \rho=w$, we obtain

$$
\frac{E}{E_{0}}=\left(\frac{a}{a_{0}}\right)^{-3 w} .
$$

Since the Hubble law is

$$
\frac{E_{\text {emitted }}}{E_{\text {observed }}}=\frac{a\left(t_{\text {observed }}\right)}{a\left(t_{\text {emitted }}\right)}
$$

and both contributions seem to be multiplicative, we get

$$
\frac{E_{\text {emitted }}}{E_{\text {observed }}}=\left(\frac{a\left(t_{\text {observed }}\right)}{a\left(t_{\text {emitted }}\right)}\right)^{1+3 w} .
$$

Hence the redshift depends on the matter equation of state. This correction to redshift $1+z=E_{\mathrm{e}} / E_{\mathrm{o}}$ may be useful in cosmology.

\section{Correction to the Distance Due to Pressure}

\subsection{The Hubble Diagram}

Recall the distance formula. Define $\chi$-coordinate by

$$
(\mathrm{d} \chi)^{2}=\frac{(\mathrm{d} r)^{2}}{1-\kappa r^{2}}, \kappa= \pm 1 .
$$

Then $\chi=\arcsin r$ if $\kappa=1$ and $\chi=\operatorname{asinh} r$ if $\kappa=-1$. Hence $r=\operatorname{sinn}(\chi)$ where

$$
\operatorname{sinn}(\chi)=\left\{\begin{array}{lll}
\sin \chi, & \kappa=1 \quad(0 \leq \chi \leq \pi), \\
\chi, & \kappa=0 & (\chi \geq 0), \\
\sinh \chi, & \kappa=-1 & (\chi \geq 0) .
\end{array}\right.
$$

The Friedmann-Robertson-Walker metric in the cosmic time becomes

$$
\begin{aligned}
(\mathrm{d} s)^{2}= & c^{2}(\mathrm{~d} t)^{2} \\
& -a(t)^{2}\left((\mathrm{~d} \chi)^{2}+\operatorname{sinn}(\chi)^{2}\left((\mathrm{~d} \theta)^{2}+\sin ^{2} \theta(\mathrm{d} \varphi)^{2}\right)\right) .
\end{aligned}
$$

The space part of the metric is

$$
(\mathrm{d} l)^{2}=a(t)^{2}\left((\mathrm{~d} \chi)^{2}+\operatorname{sinn}(\chi)^{2}\left((\mathrm{~d} \theta)^{2}+\sin ^{2} \theta(\mathrm{d} \varphi)^{2}\right)\right) .
$$

If $\theta=\theta_{0} \quad$ and $\quad \varphi=\varphi_{0} \quad$ then $\quad \mathrm{d} l=a(t) \mathrm{d} \chi \quad$ and $l=a(t) \chi$. For a photon $\mathrm{d} s=0$, therefore $c \mathrm{~d} t=a(t) \mathrm{d} \chi$ and

$$
\Delta \chi=c \int_{t_{e}}^{t_{0}} \frac{\mathrm{d} t}{a(t)}=c \int_{a_{e}}^{a_{0}} \frac{\mathrm{d} a}{\dot{a} a}=c \int_{a_{e}}^{a_{0}} \frac{\mathrm{d} a}{a^{2} H}=-\frac{c}{a_{0}} \int_{a_{e}}^{a_{0}} \frac{\mathrm{d}\left(a_{0} / a\right)}{H},
$$

where

$$
H=H_{0} \sqrt{\Omega_{0 m}\left(a_{0} / a\right)^{3}+\Omega_{\Lambda}+\Omega_{0 \kappa}\left(a_{0} / a\right)^{2}} .
$$

Now we use our main hypothesis:

$$
1+z=\left(\frac{a_{0}}{a}\right)^{1+3 w}
$$

Then

$$
\frac{a_{0}}{a}=(1+z)^{1 /(1+3 w)} .
$$

The value of $w$ is the function of $z$, therefore

$$
\mathrm{d} \frac{a_{0}}{a}=f_{w}(z) \mathrm{d} z
$$

where

$$
\begin{aligned}
f_{w}(z)= & \frac{1}{1+3 w}(1+z)^{\frac{1}{(1+3 w)}-1} \\
& -\frac{3 w_{z}^{\prime}}{(1+3 w)^{2}}(1+z)^{\frac{1}{(1+3 w)}} \ln (1+z) .
\end{aligned}
$$

Finally

$$
\Delta \chi=\frac{c}{a_{0}} \int_{0}^{z} \frac{f_{w}\left(z_{1}\right)}{H\left(z_{1}\right)} \mathrm{d} z_{1},
$$

where

$$
H(z)=H_{0} \sqrt{\Omega_{0 m}(1+z)^{3 /(1+3 w)}+\Omega_{\Lambda}+\Omega_{0 \kappa}(1+z)^{2 /(1+3 w)}}
$$

Suppose a source has an absolute luminosity $\mathcal{L}$, its luminosity (bolorimetric) distance is defined in terms of the measured flux $\mathcal{F}$

$$
d_{L}^{2}=\frac{\mathcal{L}}{4 \pi \mathcal{F}} .
$$

The measured flux is

$$
\mathcal{F}=\frac{\mathcal{L}}{4 \pi a_{0}^{2} r^{2}(1+z)^{2}},
$$

one factor of $(1+z)$ arising from the decrease in total energy due to the red shift of the energy of the individual photons, and the other factor of $(1+z)$ arising from the increased time interval between the detection of incoming photons due to the fact that two photons separated by a time $\delta t$ at emission, will be separated by a time $\delta t_{0}$ at the time of detection, where

$$
\delta t_{0} / a_{0}=\delta t / a \quad[14, \mathrm{p} . \sim 41] .
$$

hence

$$
\delta t_{0}=\delta t(1+z)
$$

and

$$
d_{L}=(1+z) a_{0} \operatorname{sinn}(\Delta \chi) .
$$

applying our main hypothesis (10) we get

$$
d_{L}=(1+z)^{1 /(1+3 w)} a_{0} \operatorname{sinn}(\Delta \chi) .
$$


recalling the definition of

$$
\Omega_{0 \kappa}=-\frac{\kappa c^{2}}{H_{0}^{2} a_{0}^{2}}
$$

we get

$$
a_{0}=\frac{c}{H_{0} \sqrt{\left|\Omega_{0 \kappa}\right|}} \text { for } \kappa= \pm 1 .
$$

therefore the luminosity distance is

$$
d_{L}=(1+z)^{\frac{1}{1+3 w}} \frac{c}{H_{0} \sqrt{\left|\Omega_{0 \kappa}\right|}} \operatorname{sinn}\left(\sqrt{\left|\Omega_{0 \kappa}\right|} \int_{0}^{z} \frac{f_{w}\left(z_{1}\right)}{H\left(z_{1}\right) / H_{0}} d z_{1}\right)
$$

We compared this model with the experimental data of the Supernova Cosmology Project Supernova Ia compilation ${ }^{1}$. In general no more than two parameters can be determined from these statistical problems [15]. We assumed that $\Omega_{0 m}=1.01$ and $\Lambda=0$, hence

$$
\Omega_{0 \kappa}=1-\Omega_{0 m}=-0.01
$$

(yet $\kappa>0$ and curvature is positive). We assumed also

$$
w(z)=v z,
$$

where $v$ is the parameter of this model. We found the optimal values

$$
v=-0.05_{ \pm 0.02}
$$

and

$$
H_{0}=66_{ \pm 5} \frac{\mathrm{km}}{\mathrm{s} \cdot \mathrm{Mps}} .
$$

the Hubble diagram with these parameters is presented on the Figure 1. The quality of this regression is as high as it is in the $\Lambda \mathrm{CDM}$ model. High values of the mass density are consistent with the experimental data on Supernova Ia within this model. Yet the value of $w$ is much less in the absolute value than in the $\Lambda \mathrm{CDM}$ model.

The Taylor expansion of the luminosity distance (18) is

$$
\begin{aligned}
d_{L}= & \frac{c}{H_{0}} z-\frac{c\left(\Omega_{0 m}+\left.12 \frac{\mathrm{d} w}{\mathrm{~d} z}\right|_{z=0}-2-2 \Omega_{\Lambda}\right)}{4 H_{0}} z^{2} \\
& +O\left(z^{3}\right)(z \rightarrow 0) .
\end{aligned}
$$

The optimal values for this simplified case for $z<0.3$ are

$$
H_{0}=69_{ \pm 2} \frac{\mathrm{km}}{\mathrm{s} \cdot \mathrm{Mps}}
$$

and

$$
\Omega_{0 m}+\left.12 \frac{\mathrm{d} w}{\mathrm{~d} z}\right|_{z=0}-2 \Omega_{\Lambda}=-0.9_{ \pm 0.4} .
$$

${ }^{1}$ http://supernova.lbl.gov/Union/figures/SCPUnion2.1_mu_vs_z.txt

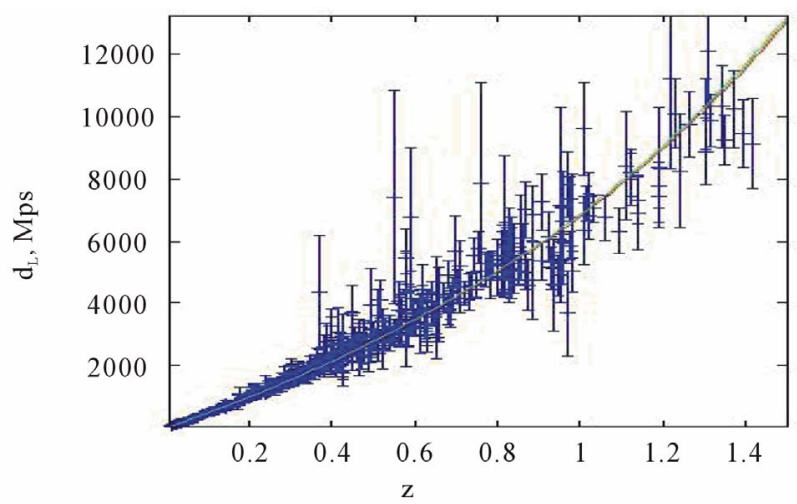

(a)

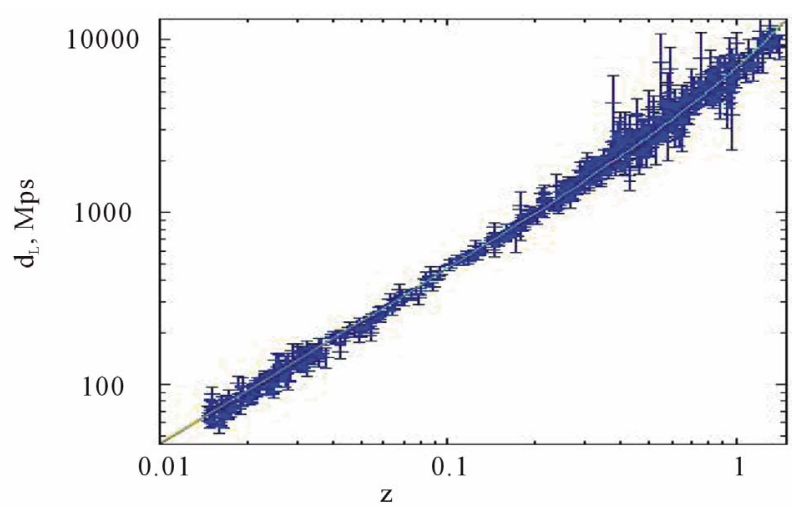

(b)

Figure 1. The hubble diagram.

\subsection{Comparing with the Riess Expansion}

In the flat Universe $\left(\Omega_{0 \kappa}=0\right)$ we can compare (20) with the Equation (10) in [16, p. 25]:

$$
d_{L}=\frac{c z}{H_{0}}\left(1+\frac{1}{2}\left(1-q_{0}\right) z+O\left(z^{2}\right)\right) .
$$

therefore the retardation parameter

$$
q_{0}=\frac{\Omega_{0 m}}{2}+\left.6 \frac{\mathrm{d} w}{\mathrm{~d} z}\right|_{z=0}-\Omega_{\Lambda}
$$

If the parameter introduced in this paper

$$
\left.\frac{\mathrm{d} w}{\mathrm{~d} z}\right|_{z=0}<-\frac{1}{6}\left(\frac{\Omega_{0 m}}{2}-\Omega_{\Lambda}\right),
$$

we obtain acceleration. Hence the acceleration of the Universe appears as a pure observational effect due to the negative pressure.

\subsection{Fate of the Universe}

Note that we have chosen $\Omega_{0 m}=1.01$ and $\Lambda=0$ while interpreting the Supernova Ia experimental data. We had to make some assumptions on these parameters because large number of unknown parameters cannot be obtained from this simple regression. Yet since experi- 
mental data are consistent with $\Omega_{0 m}=1.01$ and $\Lambda=0$, then one can say that there is a solution of the Friedman equation wich accelerates for a short time in the beginning but there is a limit for the scale factor $a$. We can smoothly glue another solution where the Universe shrinks.

The question of the fate of matter at the end of the Universe is the most complicated. We assume that the matter does not disappeared at the end of the Universe. The matter with some unknown critical density should reproduce the hydrogen and provide condition for another Big Bung. The remnants of the Big Bung are black holes. We assume that all their singularities are topologically equivalent and lead to the same space-time moment in the past where the collapsing stage of the previous Universe ends and the new Big Bung begins.

\section{The Section Curvatures}

For completeness let us review the Christoffel symbols for the Friedmann-Robertson-Walker metric (4) and the section curvatures. The Christoffel symbols for the timelike dimension $(i, j=1,2,3)$ are

$$
\Gamma_{00}^{0}=0, \Gamma_{0 j}^{i}=\Gamma_{j 0}^{i}=\frac{\dot{a}}{a} \delta_{j}^{i}, \Gamma_{i j}^{0}=-\frac{\dot{a}}{a} g_{i j} .
$$

and $\Gamma_{0 i}^{0}=0, \Gamma_{00}^{i}=0$. The Christoffel symbols for the spacelike dimensions are

$$
\begin{gathered}
\Gamma_{11}^{1}=\frac{\kappa r}{1-\kappa r^{2}}, \quad \Gamma_{22}^{1}=-r\left(1-\kappa r^{2}\right), \\
\Gamma_{33}^{1}=-r\left(1-\kappa r^{2}\right) \sin ^{2} \theta, \\
\Gamma_{12}^{2}=\Gamma_{21}^{2}=1 / r, \quad \Gamma_{33}^{2}=\frac{1}{2} \sin 2 \theta, \\
\Gamma_{13}^{3}=\Gamma_{31}^{3}=1 / r, \quad \Gamma_{23}^{3}=\Gamma_{32}^{3}=\cot \theta .
\end{gathered}
$$

these are all the nonzero components.

Let $u, v \in T_{x} N$ be linear independent vectors of the tangent space for the manifold $N$ at the point $x$. The section curvature $K_{u \wedge v}$ is defined as

$$
K_{u \wedge v}=-\frac{\langle R(u, v) v, u\rangle}{\langle u, u\rangle\langle v, v\rangle-\langle u, v\rangle^{2}},
$$

where $R$ is the Riemannian curvature map [17, p. $\sim 94]$. Compared with the Riemannian case we have changed sign in this formula because the signature of our space is Lorentzian. With local coordinates

$$
\langle R(u, v) w, z\rangle=\sum_{i, j, k, l} R_{i j k l} z^{i} w^{j} u^{k} v^{l} .
$$

Angle brackets mean scalar product. The denominator is the square of the area of the parallelogram based on the vectors $u$ and $v$. If we choose another basis in the same two-dimensional plane $\sigma$ defined by $u$ and $v$, then the section curvature does not change. Hence the value $K_{u \wedge v}$ depends on the plane $\sigma$ only. Therefore the value $K_{u \wedge v}$ is assigned $K_{\sigma}$ and is called the section curvature of the (pseudo)Riemannian manifold $N$ at the point $x$ in the two-dimensional direction $\sigma$. In local coordinates the section curvature in the direction $i-j$ is

$$
R_{i j j i} /\left(g_{i i} g_{j j}-g_{i j}^{2}\right)
$$

(no summation for $i, j$ ). The section curvature of the FRW space in the directions $0-1,0-2,0-3$ is

$$
\frac{\ddot{a}}{a} .
$$

In general for any vectors $u, v$ such as

$$
u^{0} \neq 0, u^{1}=u^{2}=u^{3}=0, \quad v^{0}=0,
$$

the section curvature $K_{u \wedge v}=\ddot{a} / a$. The section curvature of the FRW space in the directions 1-2,1-3,2-3 is

$$
\frac{\kappa+\dot{a}^{2}}{a^{2}} \text {. }
$$

Also for any vectors $v, w$ such as $v^{0}=0$ and $w^{0}=0 \quad$ (other components are arbitrary),

$$
K_{v \wedge w}=\left(\kappa+\dot{a}^{2}\right) / a^{2} .
$$

Please note that for $\Omega_{0 m}=0$ and $\Lambda=0$ the section curvatures of the spacetime at present are zero. Indeed for the directions $1-2,1-3,2-3$ the section curvatures

$$
\begin{aligned}
\frac{\kappa+\dot{a}^{2}}{a^{2}} & =\kappa / a^{2}+H^{2} \\
& =H_{0}^{2}\left(-\Omega_{\kappa}+\left(H / H_{0}\right)^{2}\right)=H_{0}^{2}\left(\Omega_{m}+\Omega_{\Lambda}\right) .
\end{aligned}
$$

It seems sensible that without matter the spacetime should be flat. The section curvatures for the directions 0 $-1,0-2,0-3$ are $\ddot{a} / a=-q H^{2}$ where $q=-\ddot{a} a / \dot{a}^{2}$ is the retardation parameter. At present time

$$
q_{0}=\frac{3 \gamma-2}{2} \Omega_{0 m}-\Omega_{\Lambda} .
$$

In the absence of matter at present for $\Lambda=0$ it will be zero also.

\section{Conclusion}

We propose new correction to the Hubble's law which can be useful in cosmology. This correction and our assumption $w(z)=v z$ produce a model which is capable to explain the acceleration of our Universe. This model is also consistent with the parameters $\Omega_{0 m}=1.01$ and $\Lambda=0$ corresponding to nearly flat and topologically closed Universe. The value $\Omega_{0 m}=1.01$ means that the expansion of the Universe will change to shrinkage yet we should observe acceleration at present. 
The assimmetry between matter and antimatter is also explained within this model. The matter does not appear from nothing at the beginning of the Universe; it is the same matter that worked at the previous cycle. The matter does not dissappear at the end of the Universe; it will just reproduce the hydrogen. The antimatter is not holes in the Dirac sea of states with negative energy; it is the matter with opposite set of quantum numbers. During the final state of the cycle (which is equally the first state of the new cycle) all properties of matter are equalized.

\section{REFERENCES}

[1] A. A. Friedmann, "Selected Works," Moscow, Nauka, 1966 (in Russian).

[2] P. Coles and F. Lucchin, "Cosmology. The Origin and Evolution of Cosmic Structure," 2nd Edition, John Wiley and Sons, 2002.

[3] P. J. E. Peebles, "Principles of Physical Cosmology," Princeton University Press, Princeton, 1993.

[4] M. Dalarsson and N. Dalarsson, "Tensor Calculus, Relativity and Cosmology," Elsevier, Amsterdam, 2005.

[5] S. Weinberg, "Gravitation and Cosmology. Principles and Applications of the General Theory of Relativity," John Wiley and Sons, Hoboken, 1972.

[6] B. Ratra and M. S. Vogeley, "Resource Letter: The Beginning and Evolution of the Universe". arXiv:0706.1565v2.

[7] R. Kessler, et al., "First-Year Sloan Digital Sky Survey-II (SDSS-II) Supernova Results: Hubble Diagram and Cos- mological Parameters". arXiv:0908.4274v1.

[8] P. Creminelli, M. A. Luty, A. Nicolis and L. Senatore, "Starting the Universe: Stable Violation of the Null Energy Condition and Non-Standard Cosmologies". arXiv:hep-th/0606090v2.

[9] L. D. Landau and E. M. Lifshits, "Course of Theoretical Physics. V. 2. The Classical Theory of Fields," Moscow, Nauka, 1988 (in Russian).

[10] L. Wolfenstein, T. G. Trippe and C.-J. Lin, "Tests of Conservation Laws," The Review of Particle Physics. urlhttp://pdg.lbl.gov.

[11] Y.-F. Cai, T. Qiu, Y.-S. Piao, M. Li and X. Zhang, "Bouncing Universe with Quintom Matter". arXiv:0704.1090v1.

[12] E. A. Bergshoeff, et al., "Cosmological D-Instantons and Cyclic Universes". arXiv:hep-th/0504011v2.

[13] D. H. Lyth, "The Primordial Curvature Perturbation in the Ekpyrotic Universe”. \{arXiv:hep-ph/0106153v3.

[14] E. W. Kolb and M. S. Turner, "The Early Universe," Addison Wesley, Boston, 1990.

[15] M. Szydlowski, A. Kurek and A. Krawiec, "Top Ten Accelerating Cosmological Models". arXiv:0604327v2.

[16] A. G. Riess, et al., "Type Ia Supernova Discoveries at $z>1$ from the Hubble Space Telescope: Evidence for Past Deceleration and Constraints on Dark Energy Evolution". arXiv:0402512v2.

[17] Y. D. Burago and V. A. Zalgaller, "Introduction in Riemannian Geometry," St.-Petersburg, Nauka, 1994 (in Russian). 